\title{
5
}

\section{Dopant Driven Electron Beam Lithography}

\author{
Timothy E. Kidd \\ Physics Department, University of Northern Iowa, Cedar Falls, IA,
}

USA

\section{Introduction}

The scanning electron microscope (SEM) can be used for far more than just obtaining images. It has a long tradition of being used to directly manipulate a sample to create various surface structures. The scanning coils within the microscope can be utilized for directing the electron beam in a controlled manner rather than simply raster across the surface as is used in imaging. By focusing the electron beam on a given area of the sample, it can be used to induce various localized changes to the surface of a material with a high degree of precision. There are several established techniques by which an electron beam can be used to create patterned structures upon a surface, the most common of which is electron beam lithography. Electron beam lithography is a multi-step process in which a sacrificial polymer layer is first deposited onto the sample that can achieve feature sizes down to ten nanometer length scales (Broers et al. 1996; Liu et al. 2002). The electron beam can also be used to locally induce or break bonds to pattern nanostructures (Mendes et al. 2004) or simply burn material away from selected areas of the sample (Egerton et al. 2004). In essentially every case, the electron beam interacts with the surface to locally alter or break chemical bonds to form patterned surface structures with very high precision.

In the present study, we have developed a new method by which the electron beam can be used to create patterned surfaces (Kidd et al. 2011). The discovery was quite by accident, occurring during some standard studies of layered dichalcogenide crystals. These highly two dimensional materials have intriguing electronic and chemical bonding characteristics which have made them of great interest for the study of novel electronic phase transitions (Wilson et al. 1975; Sipos et al. 2008) and potential use in a variety of alternative energy applications (Whittingham 1976; Kline et al. 1981; Chen et al. 2003). The systems we had chosen for study were doped crystals, as doping these materials can be used to induce dramatic changes in their electronic and chemical behaviors (Levy 1979; Friend and Yoffe 1987). The particular sample of interest at the time was $\mathrm{Cu}_{x} \mathrm{TiSe}_{2}$, owing to a recent discovery of a superconducting phase in the system in competition with the charge density wave ground state of pure $\mathrm{TiSe}_{2}$ (Morosan et al. 2006).

The SEM was being used to examine the microstructure of single crystal samples while energy dispersive $x$-ray spectrometry (EDX), via a spectrometer attached to our microscope, was used for measuring the chemical composition of the sample. These measurements were meant to do nothing more than determine the homogeneity of our samples and how much copper was successfully incorporated as a dopant. Interesting anomalies were quickly 
discovered, however. The samples had an inhomogeneous appearance in the SEM even after surface layers were removed, although EDX measurements showed the chemical composition was essentially homogenous. The EDX measurements were then taken over longer time periods, to try to detect any subtle inhomogeneity in the sample stoichiometry. These longer measurements induced further changes in the appearance of the sample, and there were signs that the copper concentration might actually be varying with the duration of the EDX measurement itself. After some time, we hypothesized that perhaps the samples were contaminated in some way, so we attempted to drive off any contamination by heating the measured crystals in a vacuum oven. This resulted in the creation of a multitude of submicron and nanoscale copper iodide crystallites upon the sample surface, except for where the EDX measurements were performed. In areas of the sample that had been exposed for long durations to the electron beam, the surface was unchanged.

Through further experimentation, the process was refined so that the size and density of the crystallites could be controlled by varying exposure dosages and the temperature at which samples were heated after exposure. The iodine was found to come from within the sample itself. Iodine is used as a catalyst for growing large single crystals of $\mathrm{TiSe}_{2}$ and other dichalcogenides, and some remains trapped in various nooks or cracks long after the samples are grown. This iodine is then released during heating and draws copper ions from within the bulk of the $\mathrm{TiSe}_{2}$ to its surface to react and form the CuI crystallites (Jaegermann et al. 1996). The electron beam radiation serves to reduce the amount of copper arriving at the surface in the exposed portions of the surface, allowing one to devise a patterned array of sub-micron structures. Unlike traditional electron beam lithography, with its required sacrificial polymer coating, no intermediate processing is required. The sample is simply exposed to the electron beam in a controlled fashion and then reacted with some quantity of a halogen gas like iodine in a simple two-step process. This lithographic technique is essentially one derived solely from controlling the mobility of the dopant ions stored within the sample to reach the surface for reaction.

\section{Experimental methods}

The $\mathrm{Cu}_{\mathrm{x}} \mathrm{TiSe}_{2}$ samples used in this study were synthesized using a technique proven for growing large single crystals of pure $\mathrm{TiSe}_{2}$ (Balchin 1976; Kidd et al. 2002). The basic process is to heat the starting elemental powders (Alfa Aesar, $>99.5 \%$ purity) with iodine as a catalyst in a sealed and evacuated silica ampoule. The original intent for these samples was to study the superconducting ground state which emerges upon doping with copper (Morosan, Zandbergen et al. 2006). In an attempt to dope the samples with a higher concentration of $\mathrm{Cu}$ than was found in the literature, the copper was included with the initial growth of $\mathrm{Ti}$, Se, and I powders in a single step growth process rather than the standard multi-step method of first growing $\mathrm{TiSe}_{2}$ powders and then later incorporating the copper dopants.

To put it mildly, high doping concentrations were not achieved using this modified singlestep method. Instead, the copper reacted strongly with iodine in the growth ampoule to form a $\mathrm{CuI}$ film which coated the surfaces of very lightly doped $\mathrm{TiSe}_{2}$ (Figure 1). The samples had a stoichiometry of $\mathrm{Cu}_{0.04} \mathrm{TiSe}_{2}$, with copper concentrations varying by \pm 0.01 as determined by EDX. This was much less than the goal of doping concentrations greater than $10 \%$, and in fact our samples showed no signs of superconductivity down to $3 \mathrm{~K}$. 

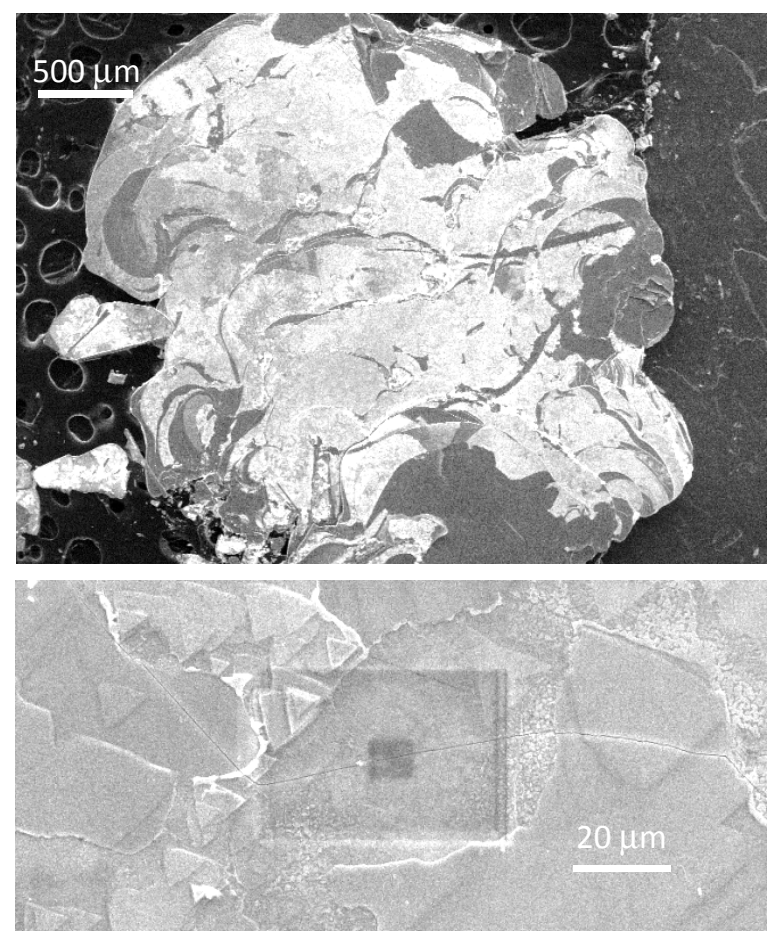

Fig. 1. SEM image of the $\mathrm{CuI}$ film coating as a grown $\mathrm{Cu}_{\mathrm{x}} \mathrm{TiSe}_{2}$ single crystal. Most of the surface is covered with a CuI film, which appears much brighter in the SEM image than surrounding $\mathrm{TiSe}_{2}$. The lower panel reveals triangular structures typical of the $\mathrm{CuI}$ coating. The darkened rectangular regions are areas in which higher magnification imaging was attempted. The focused beam burned away the $\mathrm{CuI}$ to reveal the underlying $\mathrm{TiSe}_{2}$ surface.

The CuI surface layer was not a completely uniform coating, with portions seemingly chipped away during sample handling. It appeared much brighter than uncovered areas, and typically was composed of triangular features aligned over a local area of the sample. This CuI overlayer was very sensitive to electron beam exposure. High magnification imaging would often burn away a portion or all of the CuI layer within less than a minute of imaging. Rectangular areas in which the CuI layer was thus burned away can be seen in the lower panel of Figure 1.

The SEM measurements were performed using a TESCAN Vega II microscope with an attached Bruker Quantax EDX spectrometer. SEM images and EDX data shown here were taken using $20 \mathrm{kV}$ beam voltages. The samples were manipulated in air for short periods and stored long term in a dry box. The inert $\mathrm{TiSe}_{2}$ crystals showed almost no signs of chemical decomposition months after they were grown. To expose clean portions of the samples and remove the $\mathrm{CuI}$ films, samples were exfoliated with Scotch tape to remove the uppermost surface layers. This process had to be repeated several times in some cases, as $\mathrm{CuI}$ inclusions could be found within some crystals. These inclusions, which were typically very thin CuI 
films, grew as the crystals were formed creating weak points within the crystals where exfoliation would occur but not provide a clean dichalcogenide surface.

\section{Metastable surface inhomogeneity}

SEM and EDX measurements were performed primarily on exfoliated $\mathrm{Cu}_{\mathrm{x}} \mathrm{TiSe}_{2}$ single crystals. Interestingly, even in samples which showed no evidence of $\mathrm{CuI}$ via EDX after this process, the crystals appeared inhomogeneous in SEM images as in Figure 2. The apparent brightness of the surface varied in different locations. This was quite unexpected as pure $\mathrm{TiSe}_{2}$ samples never showed any such inhomogeneity, and x-ray diffraction measurements indicated the samples were of a single phase. EDX measurements were then taken over different sections of the sample to try to correlate the apparent differences in brightness to differences in the local chemical composition. According to EDX measurements, the samples were essentially homogenous. Furthermore, high magnification SEM measurements did not reveal any substantial differences between the brighter and darker areas of the sample.

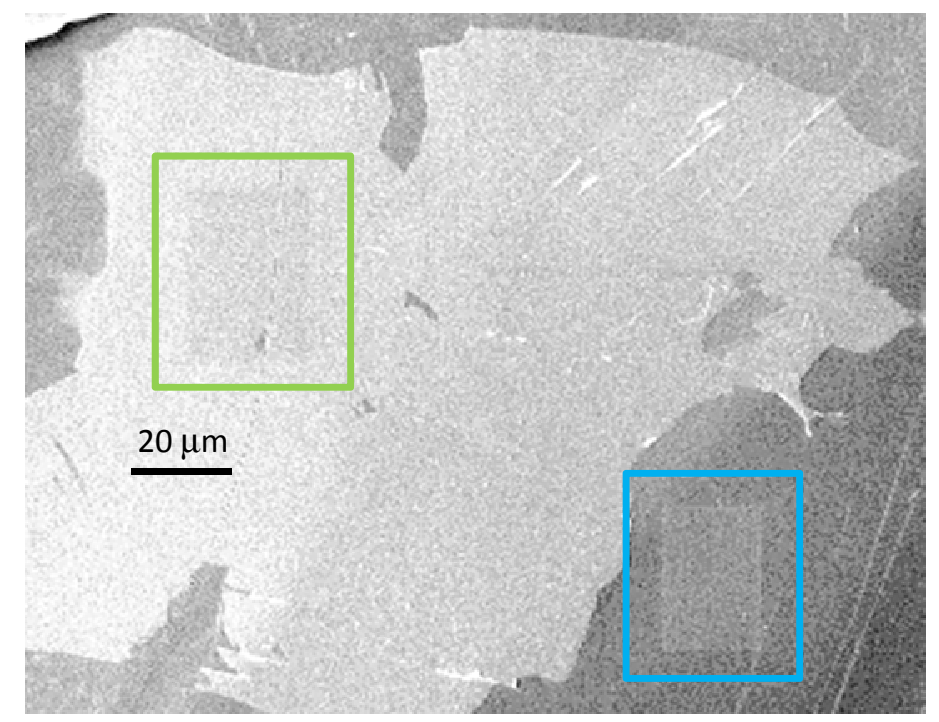

Fig. 2. SEM image taken from the inhomogeneous appearing surface of a freshly exfoliated single crystal of $\mathrm{Cu}_{0.04} \mathrm{TiSe}_{2}$. Various areas of the surface appeared more or less bright in the SEM. The colored boxes outline two areas exposed to relatively high electron beam radiation during long duration EDX measurements. The green square outlines an area from the brighter appearing portion of the surface that became darker after exposure. The blue square outlines a section from the darker area of the surface that became brighter after electron beam exposure.

To obtain more precise stoichiometry values, EDX measurements were made over longer exposure times than usual. Measurements were taken for half an hour or more, in which a relatively small portion of the surface was continuously exposed to electron beam radiation. While these measurements did not provide any conclusive results regarding the local 
stoichiometry, they did have a significant impact on the appearance of the sample surface (Figure 2). Essentially, long term electron beam exposure caused areas which initially appeared brighter to become darker and areas which were initially darker to become brighter.

The process could even be reversed to some extent in that an area could be switched back to its original appearance by a second exposure as seen in Figure 3. In this image, the sample areas appeared relatively dark until the portion in the upper half of the picture was exposed to long term electron beam radiation and became brighter. After this, a smaller portion of the sample was re-exposed to the beam. At this time, the brighter section reverted to its original appearance and the bottom half of the surface, which was not initially exposed to the beam for a long duration, was not changed at all. The edge of this second exposure is visible in the image, however. In general, it was often possible to effectively burn away a region made brighter by the beam, but we were not able to make a darkened area return to a more bright state by a second exposure.

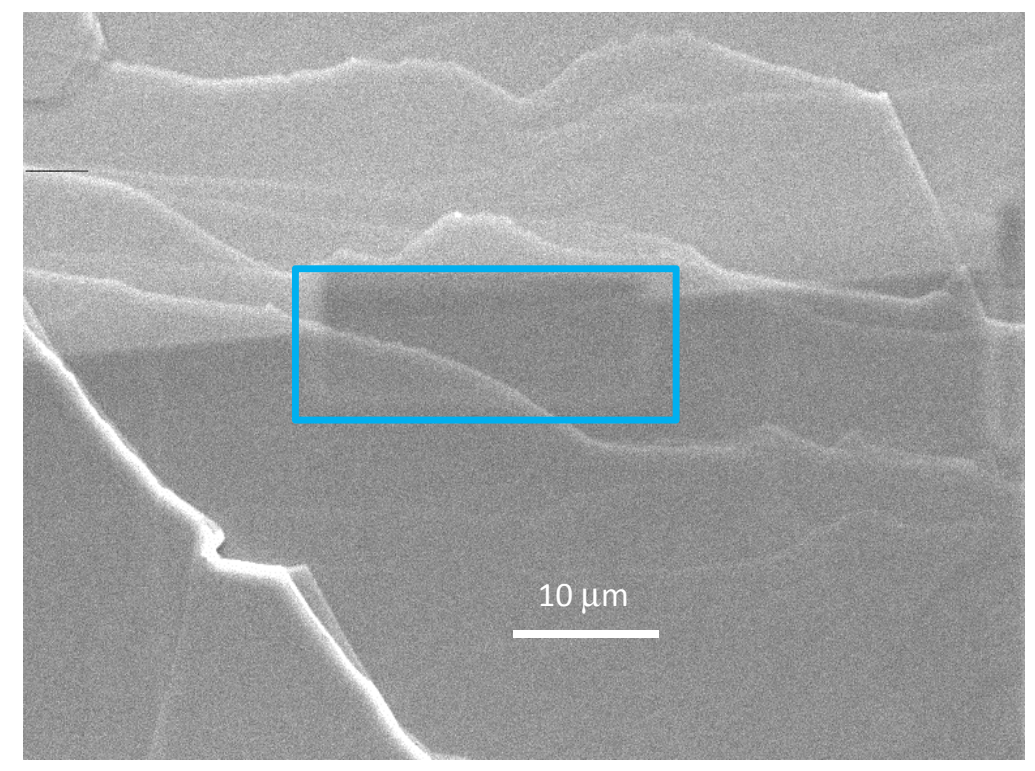

Fig. 3. SEM images of an area exposed to long term electron beam radiation multiple times. This area of the sample was originally one of the less bright regions of the surface. After long term EDX measurements, the top half of this image became brighter. A second long term EDX measurement was taken at the border between the original surface and the brightened area, as highlighted by the colored rectangle. Within this region, the area that became brighter in the initial exposure reverted to its original darker appearance after the second exposure.

These results indicate that the apparent brightness of the sample is most likely related to a phenomenon confined to the immediate surface of the sample. EDX measurements include $x$-rays generated by inelastically scattered electrons which can easily penetrate several hundred nanometers into the sample. Therefore, stoichiometry variations confined to a few surface layers are essentially undetectable by EDX. Furthermore, it is obvious that electron 
beam radiation can alter the apparent brightness of the sample as viewed in the SEM. When the beam is focused on a small area of the sample in high magnification measurements, this area would receive a relatively high dose of radiation even for short duration measurements. Therefore it is not surprising that significant differences could not be found when comparing different portions of the sample as the surface is likely changing during the measurement process itself.

These measurements in themselves show that the variation in brightness is a surface phenomenon sensitive to electron beam exposure, but do not reveal the source of the surface inhomogeneity. The apparent brightness in an SEM image can be influenced by many factors. The relative height, density, and conductivity of a given sample region can all play a role in determining how bright a particular area appears in an SEM image.

In this case, it appears that the brightness could be due to local changes in the sample density or surface conductivity. Copper ions have significant mobility within the $\mathrm{TiSe}_{2}$ solid (Gunst et al. 2000), so that any local heating or other beam interactions could potentially cause the copper ions to migrate and/or aggregate to alter the local electron density in response to beam exposure. On the other hand, insulating copper iodide films appear not only on the surface of as-grown crystals, but could also be found after exfoliations interspersed within the crystal bulk. Even though EDX measurements did not detect significant iodine levels, the brighter areas could arise from an extremely thin CuI layer. This appears to be the more likely explanation. Copper ions assume a positive oxidation state as dopants within the layers of $\mathrm{TiSe}_{2}$. If the beam caused them to migrate and cluster together in some manner, this would not be a stable configuration as the positive ions would repulse each other through Coulomb interactions to return to their equilibrium positions. The changes induced by beam exposure were stable for days if not weeks indicating equilibrium must be attained soon after the beam exposure is ended.

If the apparent changes in brightness arise to a thin $\mathrm{CuI}$ layer, there must be a source of iodine somewhere throughout the sample. In fact, a small concentration of iodine does remain interspersed throughout the sample from the growth process, where iodine is used to catalyze the growth of large single crystals. Also, iodine was found in significant amounts within cracks or at the edges of the crystals. These areas also tended to contain larger concentrations of copper, making them a mix of copper iodide and pure iodide in areas of the sample less affected by exfoliation. Due to charging effects in these insulating materials, the iodine rich particles appear much brighter than the surrounding dichalcogenide surface.

The presence of microscale iodide crystals was unexpected given the element's volatility. The samples used in this study were grown several weeks prior to the SEM measurements, making it likely that any surface iodine should have already sublimated. Therefore, the iodine seen here must have been stable while trapped inside various sample defects. This iodine would then be exposed after the exfoliation process to become more reactive.

Assuming the bright areas appear so due to a thin coating of CuI, they would become darker as this insulating material is burned away during the electron beam exposure. At the same time, the radiation from the electron beam would enhance the mobility of copper ions to flow towards the surface through local heating and/or the accumulation of negative charge. Another effect of the electron beam exposure would be to volatilize any exposed iodine. Any copper ions migrating to the surface would react with this iodine to become 
trapped as a very thin CuI layer, creating a region appearing more bright after exposure. This hypothesis is consistent with the fact that the surface was mostly stabilized within a day or so after exfoliation. While it was still possible to burn away brighter regions, it was no longer possible to reverse this process or induce darker regions to become bright on the aged samples. This is exactly what one would expect as the iodine available for reaction immediately after exfoliation would simply sublimate away so that there would be nothing left for copper ions to react with to form apparently bright regions as seen by SEM.

\section{Formation of Cul crystals}

The initial EDX measurements indicated that there could be variation in the copper concentration related to electron beam exposure, although these variations were not very significant given the precision of the measurement. If this were true, it could mean that the electron beam exposure could be used to create localized areas of superconductivity within the $\mathrm{Cu}_{\mathrm{x}} \mathrm{TiSe}_{2}$ surface and induce interesting interfacial properties between charge density wave and superconducting regions of the sample. (Morosan, Zandbergen et al. 2006; Barath et al. 2008). To better attain information about the copper distribution, it was deemed important to first remove the iodine. Copper reacts strongly with any iodine present in the system, making iodine a contaminant that can strongly affect the distribution of the dopant copper ions within the $\mathrm{TiSe}_{2}$ layers. Given the volatility of iodine, the decision was made to gently heat one of our exfoliated samples in a vacuum oven. For convenience and consistency, a sample in which EDX measurements had been performed was chosen. A low temperature, only $60^{\circ} \mathrm{C}$, was used as this should be sufficient to vaporize any exposed iodine and it was not so high as to damage the carbon tape used to mount the sample.

The results were quite surprising, as seen in Figure 4 . Not all of the iodine exposed by exfoliation sublimed from the sample during heating. In fact, it appeared as if the majority reacted with dopant copper ions to form a surface covered in CuI. That copper and iodine reacted was not completely unexpected. Halogen gas exposure has been shown to draw out high mobility dopants like copper or silver (Jaegermann, Pettenkofer et al. 1996). However, the relatively small amount of iodine seen in our measurements of the exfoliated surface did not lead us to expect the near totality with which the surface was covered after heating.

There are additional oddities concerning the copper iodine reaction of this surface. The region shown in Figure 4 is the same area as shown in Figure 3 after heat treatment. The portions of the sample that had turned relatively bright after e-beam exposure were almost completely free of $\mathrm{CuI}$ after heating. Other exposed areas can be seen as rectangular features in the image. The smaller region formed by the secondary exposure also had less $\mathrm{CuI}$ formation. However, some rectangular regions appear to have a more uniform $\mathrm{CuI}$ coverage than unexposed areas of the sample. These regions received a relatively small dose of electron beam radiation during the time in which images were taken, such as that shown in Figure 3.

The truly novel discovery was that rectangular portions of the surface remained $\mathrm{CuI}$ free. These rectangular areas could be identified as the regions on the sample which received very high electron beam exposure levels during the EDX studies. This discovery led to a more systematic series of measurements designed to illuminate whether controlled electron beam exposure could be used to create a patterned array of $\mathrm{CuI}$ features in a process similar to, but simpler than, standard electron beam lithography. 


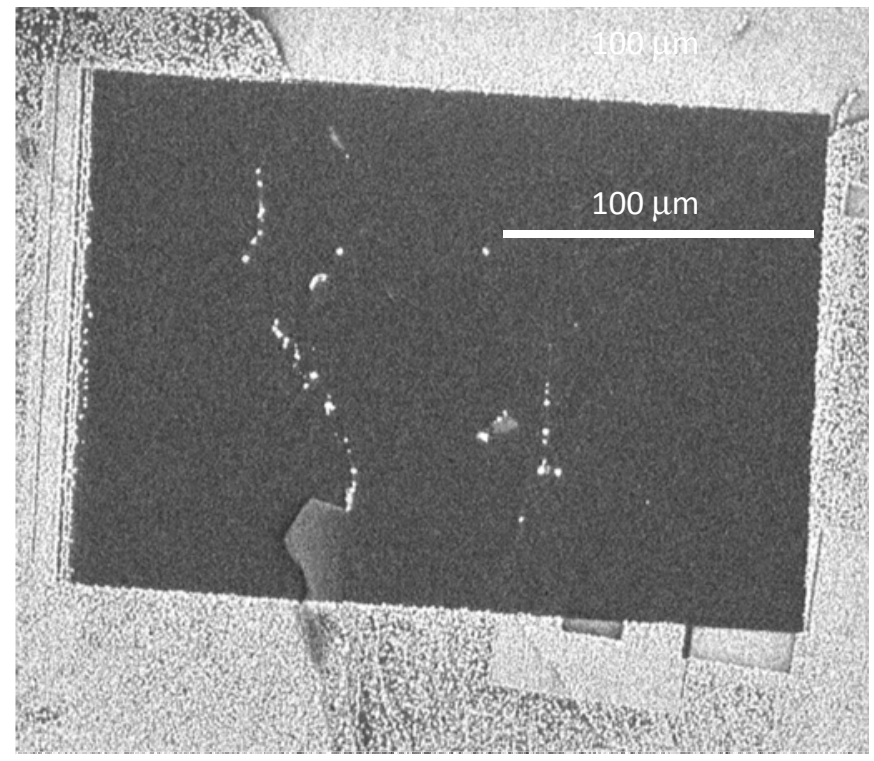

Fig. 4. SEM images taken from the area shown in Figure 3 after the sample was heated in a vacuum oven at $60^{\circ} \mathrm{C}$ for 24 hours. Iodine released from the sample during heating reacted with copper from within the sample to form CuI crystallites over the majority of the sample surface. The formation of $\mathrm{CuI}$ was reduced or eliminated in areas of the sample in which long term EDX measurements were taken.

\section{Dopant driven lithography}

To determine the effects of electron beam exposure, comparisons were made between a series of different exposure levels made upon a single $\mathrm{Cu}_{\mathrm{x}} \mathrm{TiSe}_{2}$ crystal. After the exposures were performed, the samples were transferred to the vacuum oven and heated to temperatures between $60-100^{\circ} \mathrm{C}$. The iodine exposure levels were varied by including various amounts of iodine in the vicinity of the sample when it was loaded into the vacuum oven.

Within a given sample, the results were very consistent. Figure 5 shows SEM images taken from a $\mathrm{Cu}_{x} \mathrm{TiSe}_{2}$ crystal that had been exposed to four different electron radiation doses before being heated in a vacuum oven. The surface was exposed to three ring patterns with dosages of $2.5,5$, and $10 \mathrm{mC} / \mathrm{cm}^{2}$. Near the center of each ring a small circular area was also exposed to a dosage of roughly $50 \mathrm{mC} / \mathrm{cm}^{2}$. This surface was exfoliated multiple times to attain a mostly homogenous surface to minimize any effects from a potential pre-existing $\mathrm{CuI}$ film upon the surface. These efforts were successful in that no significant changes were induced by the electron beam exposure itself. However, the radiation exposure patterns were still easily identifiable after the heat treatment. Even at low magnification levels, it can be seen that the CuI coverage is significantly reduced by the electron beam exposure. 
The influence of the electron beam exposure is more obvious at higher magnification as seen in the area exposed with a $5 \mathrm{mC} / \mathrm{cm}^{2}$ dose in Figure 6 . After heating, the surface is coated with small CuI crystallites, which are reduced in size and density in areas exposed to higher levels of electron radiation. Significant changes were difficult to detect in doses less than $1 \mathrm{mC} / \mathrm{cm}^{2}$.
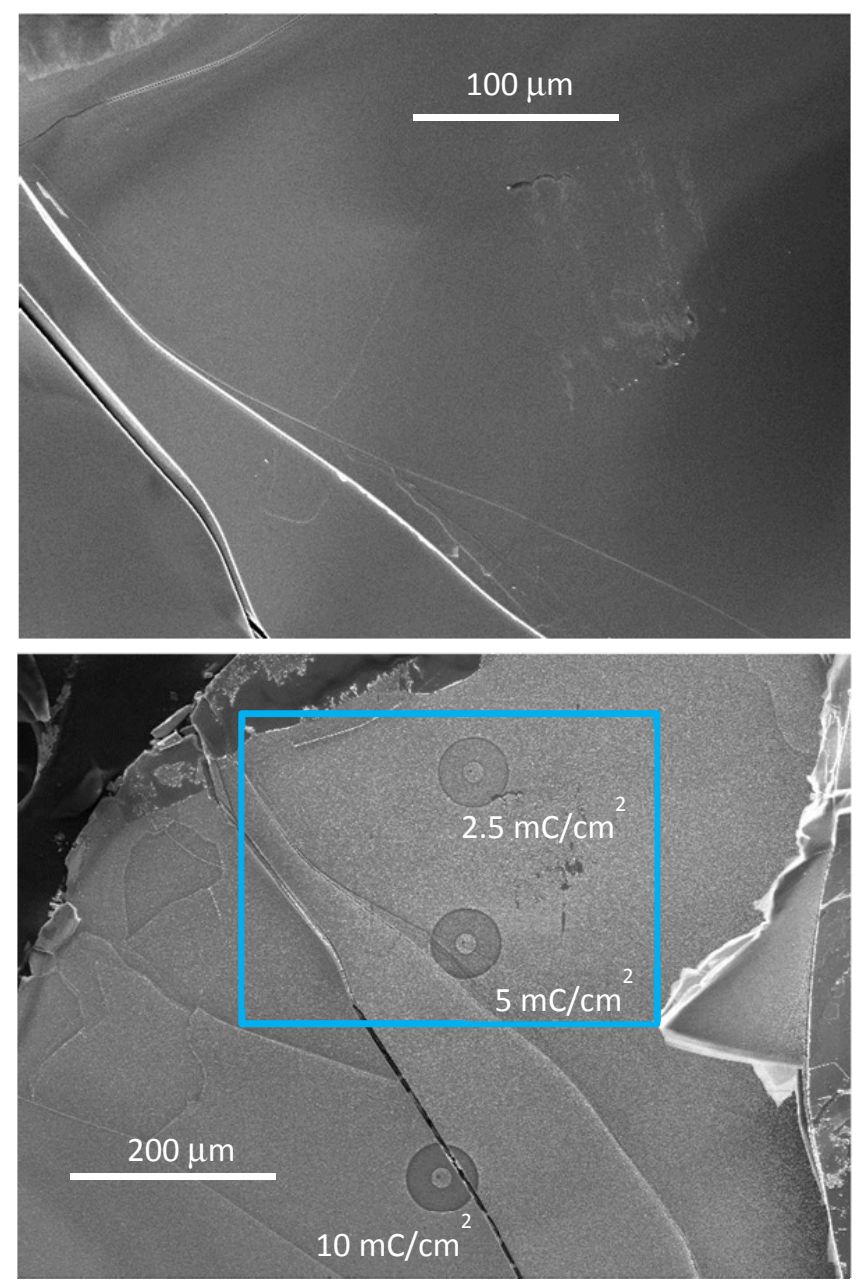

Fig. 5. SEM images before and after an irradiated sample was heated to $60^{\circ} \mathrm{C}$ in a vacuum oven for 24 hours. The top panel was taken immediately after selected areas of the surface were exposed to electron beam radiation. The surface showed no signs of damage from the electron beam exposure. The image in the bottom panel was taken after the heat treatment induced $\mathrm{CuI}$ formation. The blue rectangle indicates the area initially imaged in the upper panel, and the radiation exposure levels for each pattern are indicated on the image. Near the center of each ring, small circular areas were exposed to approximately $50 \mathrm{mC} / \mathrm{cm}^{2}$ of electron beam radiation. 

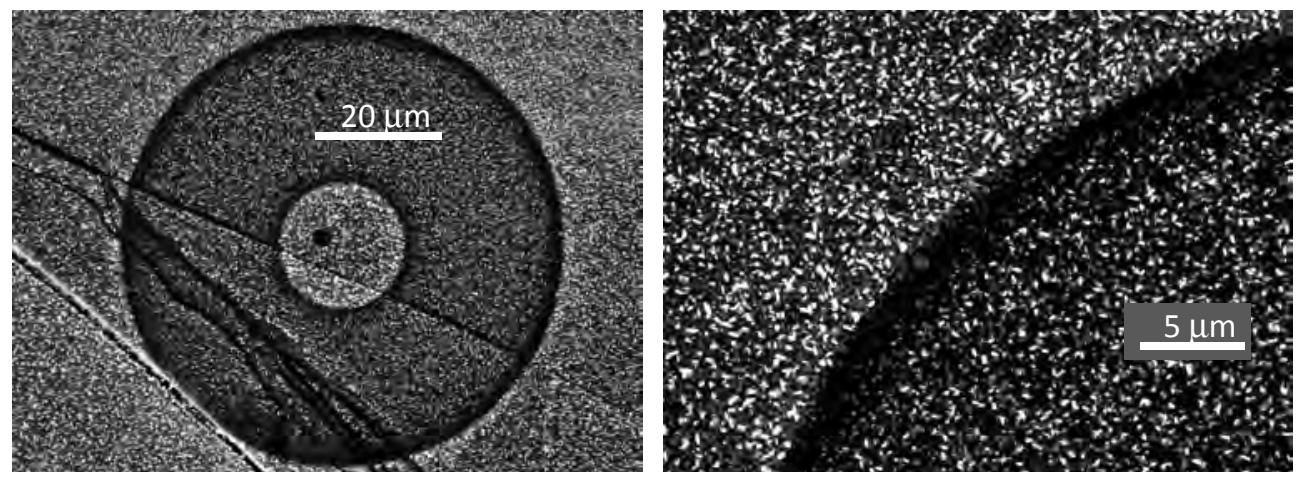

Fig. 6. SEM images taken after irradiation and vacuum heat treatment at $60^{\circ} \mathrm{C}$. The left panel is an overview of an area exposed to $10 \mathrm{mC} / \mathrm{cm}^{2}$ of radiation in a ring pattern. The right panel is a magnified view of the exterior ring edge. CuI crystallites are smaller and fewer in number in the irradiated area. The boundary region of the exposed area received a larger dose of electron radiation, resulting in even less $\mathrm{CuI}$ crystallites at the edge of the pattern.

The edges of the exposed ring were relatively dark in the SEM images, with much less CuI crystallites. This is attributed to systematic errors in the coil control program which cause the beam to linger longer at edges in the prescribed pattern. The small circle offset from the center of the ring was exposed to a much higher dosage of electron beam radiation. Through additional measurements concerning multiple samples and treatment temperatures, it was found that a dosage of $50 \mathrm{mC} / \mathrm{cm}^{2}$ was consistently sufficient to completely suppress CuI formation.

It was difficult to determine how the chemical composition of the surface was altered by various exposure doses or iodine reaction temperatures. The signal for iodine was small and copper consistently measured between three and four percent of the overall composition. Iodine could not be detected at all in areas exposed to high enough doses to completely suppress $\mathrm{CuI}$ formation, but even in these areas the copper concentration was not significantly different from the rest of the sample. These results indicate the CuI formation was confined to the surface and that the overall copper doping concentration was not substantially altered within the exposed areas.

As shown in Figure 7, the CuI crystallites are typically sub-micron structures. The size and density of these crystallites are inversely proportional to the amount of electron radiation exposure. These results show that not only can the exposure process be used to direct where the CuI crystallites are formed, but also to control their size and average spacing.

Depending on the radiation dosage and iodine reaction temperature, crystallite sizes ranged from ten nanometers to a few microns. While not particularly uniform, the size of most crystallites within a given exposure region did not deviate by more than a factor of two. The crystallite size was dependent on both the exposure level and temperature at which the iodine exposure occurred within the vacuum oven. Higher processing temperatures led to the formation of larger crystallites (Figure 8). While the smaller crystallites appeared more rounded and randomly shaped, larger structures often showed clear symmetry respective of 
an underlying crystal structure. The variation in appearance is a size-effect related phenomenon. As the size of the crystallite decreases, atoms residing at the surface represent a larger and larger percentage of the material. Surface atoms have a relatively high energy state, and when the crystallite becomes small enough, minimizing the total number of surface atoms by attaining a more rounded structure becomes more energetically favorable than maintaining the proper bonding angles representing the crystal symmetry found in larger features.

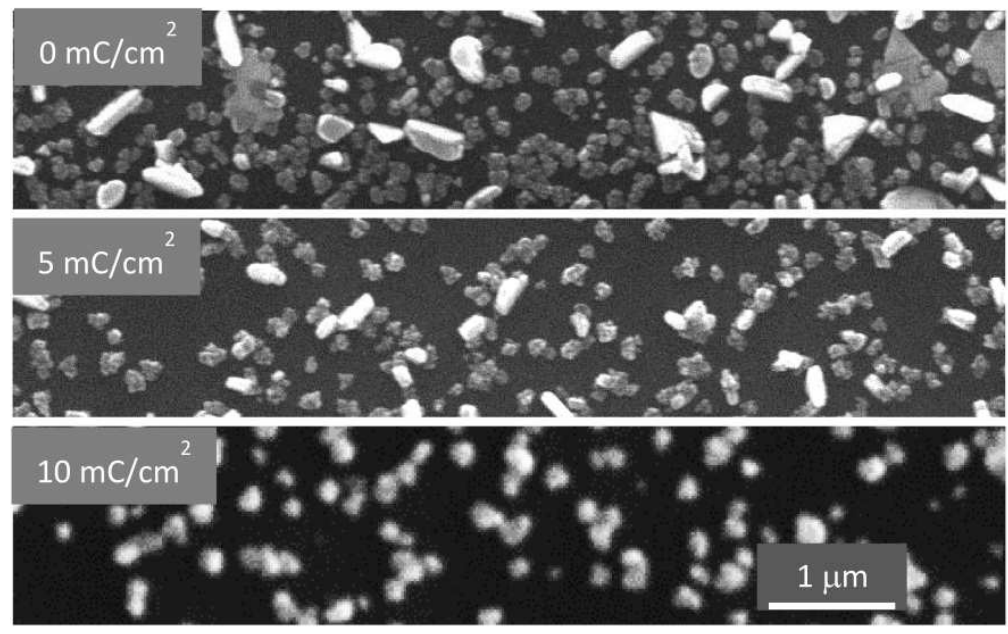

Fig. 7. SEM images comparing the effects of radiation exposure on the form of $\mathrm{CuI}$ crystallites formed after heating at $60^{\circ} \mathrm{C}$. Each panel is shown at the same scale and was taken from different areas of the same sample with the indicated radiation exposure dose.

The lateral resolution of the process is theoretically expected to be on the order of the electron beam spot used during the exposure process. However, in practice the beam spot can be made to be much smaller than the average CuI crystallite size. Thus the lateral resolution of this technique is defined more by the size of the crystallites to be obtained rather than intrinsic exposure parameters.

There were significant variations found in the size and density of crystallites formed on different $\mathrm{TiSe}_{2}$ crystals. This is attributed to variations in the amount of iodine available for reaction. It was found that including excess iodine seemed to have a much smaller effect than variations seen from sample to sample, indicating that the CuI reaction is dominated by iodine emerging from the $\mathrm{TiSe}_{2}$ sample itself. While iodine is a volatile element in ambient conditions, it can remain present for years trapped between layers of inside defects of a $\mathrm{TiSe}_{2}$ crystal. When the sample is exfoliated, trapped iodine becomes available for reaction, especially when the sample is heated, even at temperatures below $100^{\circ} \mathrm{C}$. Iodine loaded in proximity to the crystal likely becomes volatile and spreads quickly throughout the vacuum oven, minimizing its effectiveness in forming CuI. Iodine trapped in the nooks and crannies of the sample will take more time to fully volatilize, and be in closer proximity to the surface, giving it more time to react with the copper dopant ions before it dissipates away from the sample. This hypothesis also supports the relationship between crystallite 
size and reaction temperature. At higher temperatures, the copper ions have a higher mobility which allows a larger number to reach the surface in a given time. As there is likely a limit to how long the chemical reaction takes place, heating to a higher temperature would allow more copper ions to take part in the reaction to form larger structures.

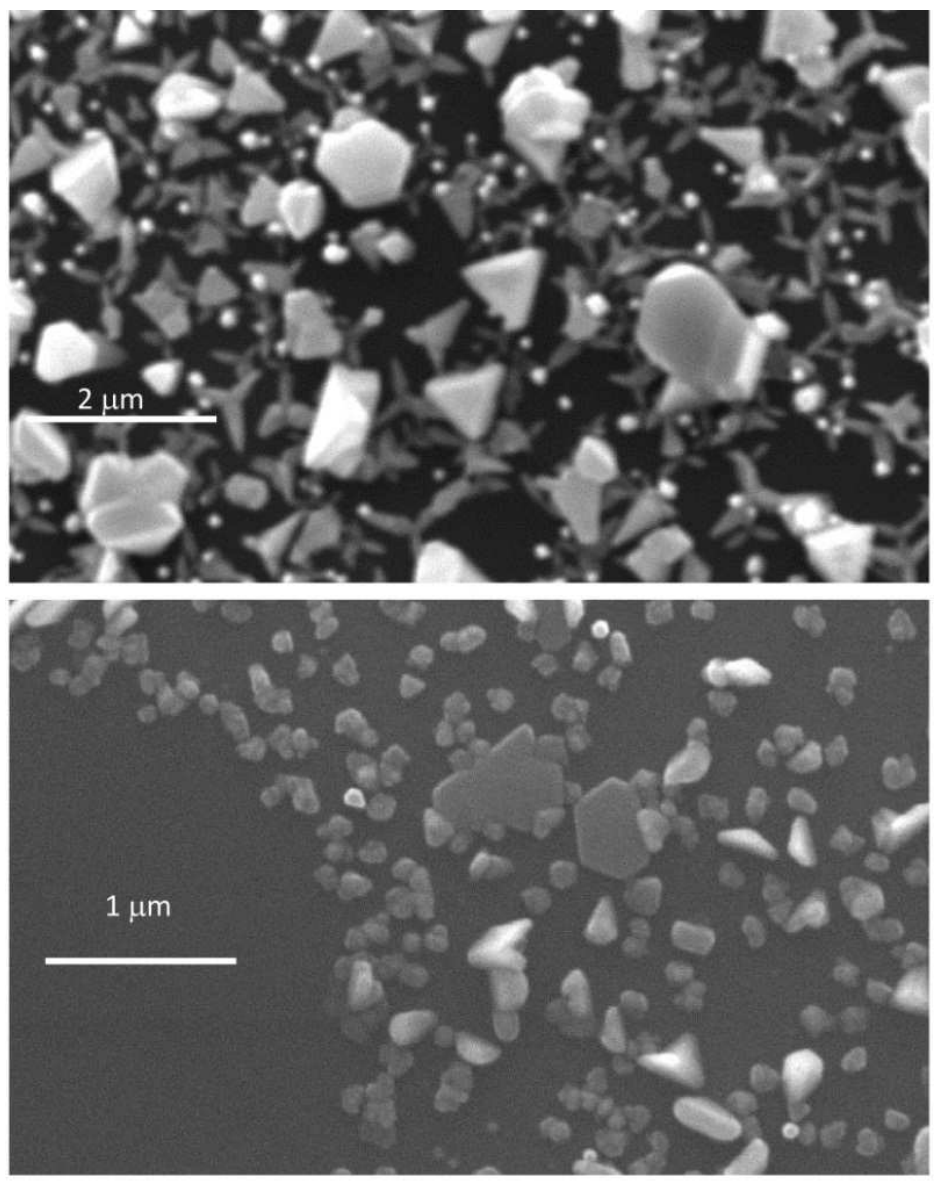

Fig. 8. SEM images comparing the effects of reaction temperature on the size of CuI crystallites. The top panel was heated in the vacuum oven at $100^{\circ} \mathrm{C}$. The bottom panel was heated to $60^{\circ} \mathrm{C}$. The bare area on the left of the bottom panel was initially dosed with about $50 \mathrm{mC} / \mathrm{cm}^{2}$ of electron beam radiation, completely suppressing the formation of $\mathrm{CuI}$.

It was also found that $\mathrm{CuI}$ formation was inhibited for samples which had been exfoliated for many days prior to beam exposure. Even though the samples looked almost identical to fresh surfaces in the SEM, including the presence of trapped iodine, CuI crystallites would rarely grow upon these aged samples. These results were reminiscent of an AFM study in which the copper ions could be drawn to the surface of a dichalcogenide crystal, but only for freshly exfoliated surfaces (Gunst, Klein et al. 2000). 


\section{Conclusions and future directions}

The experimental results show that it is possible to use electron beam exposure to control the formation of $\mathrm{CuI}$ crystallites on the surface of a $\mathrm{TiSe}_{2}$ crystal. This is a two-step process in which a fresh surface is first exposed to electron beam radiation in a controlled fashion, and then heated in the presence of iodine. Due to inconsistencies between samples, exact empirical parameters concerning the size and density of $\mathrm{CuI}$ formation have not been determined as yet, although qualitative relationships have been established. The size and density of CuI crystallites formed in this process are inversely proportional to the amount of electron beam radiation. The formation of crystallites can be completely eliminated in areas receiving relatively high exposure levels. It is evident this process can be used to create an array of CuI crystallites with diameters sufficiently small to be considered nanoparticles at certain processing conditions. The lateral resolution is essentially limited by the size of the crystallites, making this technique suitable for forming a pattern array of nanocrystallites with nanoscale precision.

In itself, this if of interest as $\mathrm{CuI}$ nanoparticles show some promise for applications in dyesensitized solar cells (Perera and Tennakone 2003) or gas sensing applications for CO and NO (Wolpert et al. 2009). The process might also be useful for controlling the local copper dopant concentration, important for exploring competition between superconducting and charge density wave ground states in the material. However, the technique would be of far greater importance if it could be extended to other dopants and/or dichalcogenide crystals. For this to occur, one must have a better understanding of the process itself. While a complete quantitative understanding remains elusive given inconsistencies between samples, a qualitative explanation is forthcoming from our observations.

The second step of the process is relatively straightforward. Copper ions have a high mobility within dichalcogenides and can be reversibly incorporated into their structure using electrical or chemical gradients.(Gunst, Klein et al. 2000) Iodine present at the sample surface will react with surface copper ions, leading to a concentration gradient near the surface. Copper ions from deeper within the sample will be driven to the surface layers by this depletion, and thus in turn react themselves. This process will continue until the iodine is depleted, the concentration gradient is reduced below some threshold value depending on the overall percentage of copper ions in the sample, or the surface is completely coated with CuI. The increased size of the CuI crystallites at higher reaction temperatures could be due to the increased mobility of $\mathrm{Cu}$ ions at higher temperatures or the fact that more iodine is released from the sample. Either process could lead to the formation of more CuI at the surface before the iodine dissipates away from the sample.

The first step of the process is perhaps not as intuitive. It was not initially clear why exposure of the sample could be used to influence the reaction of copper and iodine at the sample surface. The exposure must in some way be inhibiting the copper ions from traveling to the surface during the iodine reaction. This is most likely due to the electron beam radiation inducing a localized surface chemical reaction. When $\mathrm{TiSe}_{2}$ is exposed to wet or humid environments it will oxidize at the surface. Titanium diselenide is composed of molecular $\mathrm{TiSe}_{2}$ layers as shown in Figure 8. Hydrogen from the water will react with Se to form gaseous $\mathrm{H}_{2} \mathrm{Se}$ leaving the exposed titanium atoms to react with the remaining oxygen to form titanium oxide. Owing to the inert nature of the material and its layered structure, it 
can take days or even weeks for a visible oxide layer to be seen. However, it would not be surprising for the uppermost molecular layer to oxidize within even a few hours of exposure to ambient conditions. A surface layer of titanium oxide would be an effective barrier to $\mathrm{Cu}$ ion migration to the surface, which would explain why CuI would not form on the surface of samples that had been exfoliated for several days before electron beam exposure was initiated.

Within the SEM, the sample resides in a vacuum. However, the samples are exfoliated in air before being inserted into the microscope. This means that there will be a residual water layer of some thickness on the sample even after the measurement chamber is evacuated. In truth, such a water layer coats the entire interior surface of this and any other SEM that is not heated to at least $100^{\circ} \mathrm{C}$ before measurement. This minor amount of water would normally react very slowly with the $\mathrm{TiSe}_{2}$ surface. However, the energy provided by the electron beam radiation could easily break up the water molecules into various radical ions that would be much more reactive. This would in turn create a titanium oxide surface layer to inhibit copper ion migration. The uniformity and thickness of such an oxide layer would depend on the radiation exposure level, making it possible to fine tune how much copper ions migrate to the surface during the time in which the sample reacts with iodine in the second step of the process. It is true that other chemical reactions could occur on the surface, such as with remnant gas molecules within the SEM, however the low pressure $\left(<10^{-2} \mathrm{~Pa}\right)$ makes this unlikely.

These results make it apparent which systems could be best utilized with this technique. A wide range of ions and molecules can be reversibly stored within various dichalcogenides (Levy 1979). This makes a wide range of surface features possible, given the proper method by which materials could be induced to leave the sample. The most straightforward extension of this technique would be to utilize silver rather than copper ions. Silver and copper both have similar mobility within a given dichalcogenide and silver also is highly reactive with halogen gasses. Thus it should not be difficult to create a patterned array of silver iodide crystallites on the surface of $\mathrm{TiSe}_{2}$. AgI has been used in photographic and other optical applications for decades, and an array of AgI nanocrystallites could easily be reduced to form silver nanoparticles which are of high interest for their antibacterial properties.

Current research is being directed towards refining this technique to enable the formation of various copper and silver halide nanoparticle arrays. Titanium diselenide is used as the principle host, but Ta based dichalcogenides are also of interest for their similar chemical characteristics. It is hoped that this technique can be coupled with traditional electron beam and/or scanning probe lithographic techniques to enable to the fabrication of prototype device structures.

\section{Acknowledgements}

The author would like to thank Dusty Klein and Tyler Rash for assisting with the SEM/EDX measurements and Dr. Laura Strauss for assisting with crystal growth. This work was supported by Battelle and the Iowa Office of Energy Independence grant \#09-IPF-11. The author was also supported by a UNI summer fellowship during portions of this research. 


\section{References}

Balchin, A. A. (1976). Growth and the Crystal Characteristics of Dichalcogenides Having Layer Structures. Crystallography and Crystal Chemistry of Materials with Layered Structures. F. Levy. Dordrecht, D. Reidel. 2: 1-50.

Barath, H., M. Kim, J. F. Karpus, S. L. Cooper, P. Abbamonte, E. Fradkin, E. Morosan and R. J. Cava (2008). "Quantum and Classical Mode Softening Near the Charge-DensityWave\&\#150;Superconductor Transition of Cu_\{x\}TiSe_\{2\}." Physical Review Letters 100(10): 106402.

Broers, A. N., A. C. F. Hoole and J. M. Ryan (1996). "Electron beam lithography--Resolution limits." Microelectronic Engineering 32(1-4): 131-142.

Chen, J., S.-L. Li, Z.-L. Tao, Y.-T. Shen and C.-X. Cui (2003). "Titanium Disulfide Nanotubes as Hydrogen-Storage Materials." Journal of the American Chemical Society 125(18): 5284-5285.

Egerton, R. F., P. Li and M. Malac (2004). "Radiation damage in the TEM and SEM." Micron 35(6): 399-409.

Friend, R. H. and A. D. Yoffe (1987). "Electronic properties of intercalation complexes of the transition metal dichalcogenides." Advances in Physics 36(1): 1.

Gunst, S., A. Klein, W. Jaegermann, Y. Tomm, H. Crawack and H. Jungblut (2000). "Intercalation and deintercalation of transition metal dichalcogenides: Nanostructuring of intercalated phases by scanning probe microscopy." Ionics 6(3): 180-186.

Jaegermann, W., C. Pettenkofer, O. Henrion, Y. Tomm, C. Papageorgopoulos, M. Kamaratos and D. Papageorgopoulos (1996). "Surface science investigations of $\mathrm{Cu}$ intercalation in $1 \mathrm{~T} \mathrm{TaSe} 2$ and $\mathrm{TiSe}_{2}$ and its deintercalation by adsorbed $\mathrm{Br}_{2}$." Ionics 2(3): 201-207.

Kidd, T. E., D. Klein, T. A. Rash and L. H. Strauss (2011). "Dopant based electron beam lithography in CuxTiSe2." Applied Surface Science 257(8): 3812-3816.

Kidd, T. E., T. Miller, M. Y. Chou and T. C. Chiang (2002). "Electron-Hole Coupling and the Charge Density Wave Transition in TiSe2." Physical Review Letters 88(22): 226402.

Kline, G., K. Kam, D. Canfield and B. A. Parkinson (1981). "Efficient and stable photoelectrochemical cells constructed with WSe2 and MoSe2 photoanodes." Solar Energy Materials 4(3): 301-308.

Levy, F., Ed. (1979). Intercalated Layer Materials. Physics and Chemistry of Materials with Layered Structures. Dordrecht, D. Reidel.

Liu, K., P. Avouris, J. Bucchignano, R. Martel, S. Sun and J. Michl (2002). "Simple fabrication scheme for sub-10 nm electrode gaps using electron-beam lithography." Applied Physics Letters 80(5): 865-867.

Mendes, P. M., S. Jacke, K. Critchley, J. Plaza, Y. Chen, K. Nikitin, R. E. Palmer, J. A. Preece, S. D. Evans and D. Fitzmaurice (2004). "Gold Nanoparticle Patterning of Silicon Wafers Using Chemical e-Beam Lithography." Langmuir 20(9): 3766-3768.

Morosan, E., H. W. Zandbergen, B. S. Dennis, J. W. G. Bos, Y. Onose, T. Klimczuk, A. P. Ramirez, N. P. Ong and R. J. Cava (2006). "Superconductivity in CuxTiSe2." Nature Physics 2(8): 544-550.

Perera, V. P. S. and K. Tennakone (2003). "Recombination processes in dye-sensitized solidstate solar cells with $\mathrm{CuI}$ as the hole collector." Solar Energy Materials and Solar Cells 79(2): 249-255. 
Sipos, B., A. F. Kusmartseva, A. Akrap, H. Berger, L. Forro and E. Tutis (2008). "From Mott state to superconductivity in 1T-TaS2." Nature Materials 7(12): 960-965.

Whittingham, M. S. (1976). "Electrical Energy Storage and Intercalation Chemistry." Science 192(4244): 1126-1127.

Wilson, J. A., F. J. D. Salvo and S. Mahajan (1975). "Charge-density waves and superlattices in the metallic layered transition metal dichalcogenides." Advances in Physics 24(2): 117-201.

Wolpert, B., O. S. Wolfbeis and V. M. Mirsky (2009). "Gas sensing properties of electrically conductive $\mathrm{Cu}(\mathrm{I})$ compounds at elevated temperatures." Sensors and Actuators B: Chemical 142(2): 446-450. 


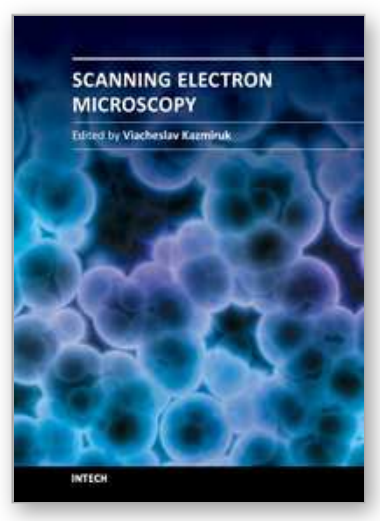

\author{
Scanning Electron Microscopy \\ Edited by Dr. Viacheslav Kazmiruk
}

ISBN 978-953-51-0092-8

Hard cover, 830 pages

Publisher InTech

Published online 09, March, 2012

Published in print edition March, 2012

Today, an individual would be hard-pressed to find any science field that does not employ methods and instruments based on the use of fine focused electron and ion beams. Well instrumented and supplemented with advanced methods and techniques, SEMs provide possibilities not only of surface imaging but quantitative measurement of object topologies, local electrophysical characteristics of semiconductor structures and performing elemental analysis. Moreover, a fine focused e-beam is widely used for the creation of micro and nanostructures. The book's approach covers both theoretical and practical issues related to scanning electron microscopy. The book has 41 chapters, divided into six sections: Instrumentation, Methodology, Biology, Medicine, Material Science, Nanostructured Materials for Electronic Industry, Thin Films, Membranes, Ceramic, Geoscience, and Mineralogy. Each chapter, written by different authors, is a complete work which presupposes that readers have some background knowledge on the subject.

\title{
How to reference
}

In order to correctly reference this scholarly work, feel free to copy and paste the following:

Timothy E. Kidd (2012). Dopant Driven Electron Beam Lithography, Scanning Electron Microscopy, Dr. Viacheslav Kazmiruk (Ed.), ISBN: 978-953-51-0092-8, InTech, Available from:

http://www.intechopen.com/books/scanning-electron-microscopy/dopant-driven-electron-beam-lithography

\section{INTECH}

open science | open minds

\author{
InTech Europe \\ University Campus STeP Ri \\ Slavka Krautzeka 83/A \\ 51000 Rijeka, Croatia \\ Phone: +385 (51) 770447 \\ Fax: +385 (51) 686166 \\ www.intechopen.com
}

\author{
InTech China \\ Unit 405, Office Block, Hotel Equatorial Shanghai \\ No.65, Yan An Road (West), Shanghai, 200040, China \\ 中国上海市延安西路65号上海国际贵都大饭店办公楼 405 单元 \\ Phone: +86-21-62489820 \\ Fax: +86-21-62489821
}


(C) 2012 The Author(s). Licensee IntechOpen. This is an open access article distributed under the terms of the Creative Commons Attribution 3.0 License, which permits unrestricted use, distribution, and reproduction in any medium, provided the original work is properly cited. 